\title{
Virtual Reality tremor reduction in Parkinson's disease
}

John Cornacchioli, Alec Galambos, Stamatina Rentouli, Robert Canciello, Roberta Marongiu, Daniel Cabrera, eMalick G. Njie*

NeuroStorm, Inc, New York City, New York, United States of America stoPD, Long Island City, New York, United States of America

${ }^{*}$ Corresponding author at: Dr. eMalick G. Njie, NeuroStorm, Inc, 1412 Broadway, FI21, New York, NY 10018. Tel.: +1 347651 1267; emalick.njie@gmail.com, emalick@neurostorm.studio

\begin{abstract}
:
Multidisciplinary neurotechnology holds the promise of understanding and non-invasively treating neurodegenerative diseases. In this preclinical trial on Parkinson's disease (PD), we combined neuroscience together with the nascent field of medical virtual reality and generated several important observations. First, we established the Oculus Rift virtual reality system as a potent measurement device for parkinsonian involuntary hand tremors (IHT). Interestingly, we determined changes in rotation were the most sensitive marker of PD IHT. Secondly, we determined parkinsonian tremors can be abolished in VR with algorithms that remove tremors from patients' digital hands. We also found that PD patients were interested in and were readily able to use VR hardware and software. Together these data suggest PD patients can enter VR and be asymptotic of PD IHT. Importantly, VR is an open-medium where patients can perform actions, activities, and functions that positively impact their real lives - for instance, one can sign tax return documents in VR and have them printed on real paper or directly e-sign via internet to government tax agencies. Lastly, we generated a technical framework wherein movements in the real world can be measured side-by-side with those in virtual reality. With this framework, we observed anecdotal evidence of parkinsonian tremors being reduced in real life when our algorithms abolished digital hand tremors in VR.
\end{abstract}

\section{Key Words:}

Neurodegeneration, Parkinson's disease, Essential Tremor, Deep Brain Stimulation, Levodopa, Virtual Reality, Oculus Rift, Medical Virtual Reality, Tremor stabilization, Equalizers

\section{Contributions:}

EGN conceived and directed the experimental paradigm. JC generated the tremor stabilization algorithms. JC, AG and RC generated the virtual reality environments. RM and stoPD coordinated study participants. EGN, JC and AG performed the experiments. DC generated the patient data collection forms. EGN analyzed the data. EGN wrote the manuscript with JC, AC, and SR.

\section{Funding:}

This study was funded by Dr. eMalick. G. Njie.

\section{Introduction:}

Parkinson's disease (PD) is a neurodegenerative disease affecting approximately $1 \%$ of adults worldwide over age 60 including one million Americans (Pringsheim et al., 2014; Marras et al., 2018). The disease is characterized by dopaminergic neuronal loss in the central nervous system that results in peripheral motor symptoms. In particular, most PD patients suffer $4-7 \mathrm{~Hz}$ involuntary hand tremors $(\mathrm{IHT})(\mathrm{H}$. J. Lee 
et al., 2016) causing significant disability in Activities of Daily Living (ADL) (S. Y. Lee et al., 2016). Currently, there is no cure for PD. However, several treatments are available to patients. These include dopaminergic drugs which counter the neuronal cell loss associated with dopamine depletion (Salat and Tolosa, 2013). Another treatment is Deep Brain Stimulation (DBS), a type of brain surgery in which microelectrodes are inserted into regions such as the subthalamic nucleus to provide pulses of electricity (Benabid et al., 1994; Weaver et al., 2009) which are thought to suppress pathological synchronization of neurons (Eusebio et al., 2011). These treatments are effective but temporary and carry a significant risk of physiological and cognitive side-effects (Cools et al., 2003; Cyron, 2016; Hickey and Stacy, 2016; Buhmann et al., 2017).

Virtual Reality (VR) is an emerging technology where the visual field of users is fully replaced with a three-dimensional digital representation. The malleability of this representation presents a significant opportunity for understanding and treating Parkinson's disease. For instance, PD patients had improved gait and suffered fewer falls after being trained in VR obstacle courses (Mirelman et al., 2016). Unfortunately, little further work has been done to innovate VR for the benefit of PD patients.

Towards this, we examined the potential of VR to ameliorate the motor symptoms of PD. VR controllers are sensitive instruments that can measure position in space over time with significant precision. With these controllers, we have captured high-fidelity profiles of patient tremors. We then developed algorithms that cancel out tremors in the virtual space by determining the expected centroid of hand position in real-time and generating a tremor-free digital representation of patients' hands in VR thus enabling patients to be asymptomatic of IHT in VR.

Interestingly, PD is thought to be a synchronization disease where groups of neurons progressively enter into pathological synchronous patterns of firings (Wingeier et al., 2006; Kühn et al., 2008; Nabi et al., 2013; Wilson and Moehlis, 2015). This theory predicts that interventions that break pathological synchrony will reduce IHT. For instance, closed-loop and coordinated-reset DBS in which an anti-signal of electrical energy is pulsed into pallidal or thalamic nuclei reduced coherence of oscillating neurons and had a therapeutic effect on tremors (Rosin et al., 2011; Little et al., 2013; Adamchic et al., 2014). Recently, coordinated-reset vibrotactile finger movement reduced IHT (Syrkin-Nikolau et al., 2018), suggesting that mechanosensory (and possibly other) modalities can effectively provide a therapeutic anti-signal. We therefore hypothesize that manipulating the visual field in VR such that patients' digitallyrepresented hands lack IHT could also provide a therapeutic anti-signal. We describe in the following a preclinical trial testing this assertion.

\begin{tabular}{|c|c|c|c|c|}
\hline Individual & Gender & Age of PD onset & Duration of diagnosis & IHT (5 is most severe) \\
\hline 1 & $\mathrm{~F}$ & Late onset & 4 years & 4 \\
\hline 2 & $M$ & Early onset & 2 years & 2 \\
\hline 3 & M & Late onset & 8 years & 1 \\
\hline 4 & $\mathrm{~F}$ & Late onset & 2 years & 4 \\
\hline 5 & M & Early onset & 5 years & 3 \\
\hline 6 & $M$ & Late onset & 3 years & 1 \\
\hline 7 & $\mathrm{n} / \mathrm{a}$ & Late onset & 2 years & $\mathrm{n} / \mathrm{a}$ \\
\hline 8 & $\mathrm{~F}$ & Late onset & 2 years & 3 \\
\hline 9 & $\mathrm{n} / \mathrm{a}$ & Late onset & $\mathrm{n} / \mathrm{a}$ & $\mathrm{n} / \mathrm{a}$ \\
\hline 10 & $\mathrm{~F}$ & control & & \\
\hline
\end{tabular}

Table 1. Demographic information of study participants. Note patients were not screened for prognosis and severity of PD, or whether on oral or surgical medication. Early onset defined by diagnoses $<=50$ years. Severity of involuntary hand tremor (IHT) scale ranges from 1 (no tremors) to 5 (severe tremors). All data was self-reported.

\section{Results:}

The Oculus Rift primarily uses accelerometers and gyroscopes embedded within its Touch controllers to determine physical position in space. These record changes of rotational angles $\alpha, \beta, \gamma$, and Cartesian axes $X, Y, Z$ at up to $1000 \mathrm{~Hz}$. However, we recorded at $4 \mathrm{~Hz}$ which is in the range of frequencies $\mathrm{PD}$ tremors occur at $(\mathrm{H}$. J. Lee et al., 2016). We recruited ten volunteer participants for observation of PD 
associated IHT (Table 1). Patients were placed in a virtual kitchen where they could perform tasks such as placing bread into a toaster and pouring cereal into a bowl. This familiarized the patients with the Oculus headset and its Touch controllers. We then loaded a bare-bones virtual testing environment and instructed patients to perform a postural tremor test (PTT) in which the patients stabilize their backs against a wall and position their hands in the air against gravity for two minutes. This position elicits parkinsonian tremors as it places the patients' hands in a posture such that they must maintain gait against gravity (Koller, Vetere-Overfield and Barter, 1989). The PTT captures distinguishing features of parkinsonian tremors such as reduced movement with conscious activity and exacerbated movement with rest.

During the PTT, Tremor Stabilization Algorithms (TSA; see Methods section for algorithm description) were either turned off or turned on (TSA-OFF, TSA-ON). Specifically, one minute of the PTT was TSA-OFF and one minute was TSA-ON (Figure 1). We found that the rotational signature of IHT was most discernible with tremor-associated changes in hand rotation (Figure $1 \mathrm{~A}-\mathrm{C}$ ) compared to changes in hand position (Figure 1D-F). Importantly, we recorded the positional information of patients' hands in the real world (i.e., the position of the Oculus Touch controllers in physical space) simultaneously with the virtual position of the patients' digital hands in VR. This enabled segregation of effects on IHT in the real world from those in VR. As describing results in physical space and virtual space can easily become confusing: we define data of hand positions in physical space as classical reality (CR, green traces in Figure 1 \& Figure 3) and data of digital hand positions in virtual space as virtual reality (VR, yellow traces in Figure 1 \& Figure 2).

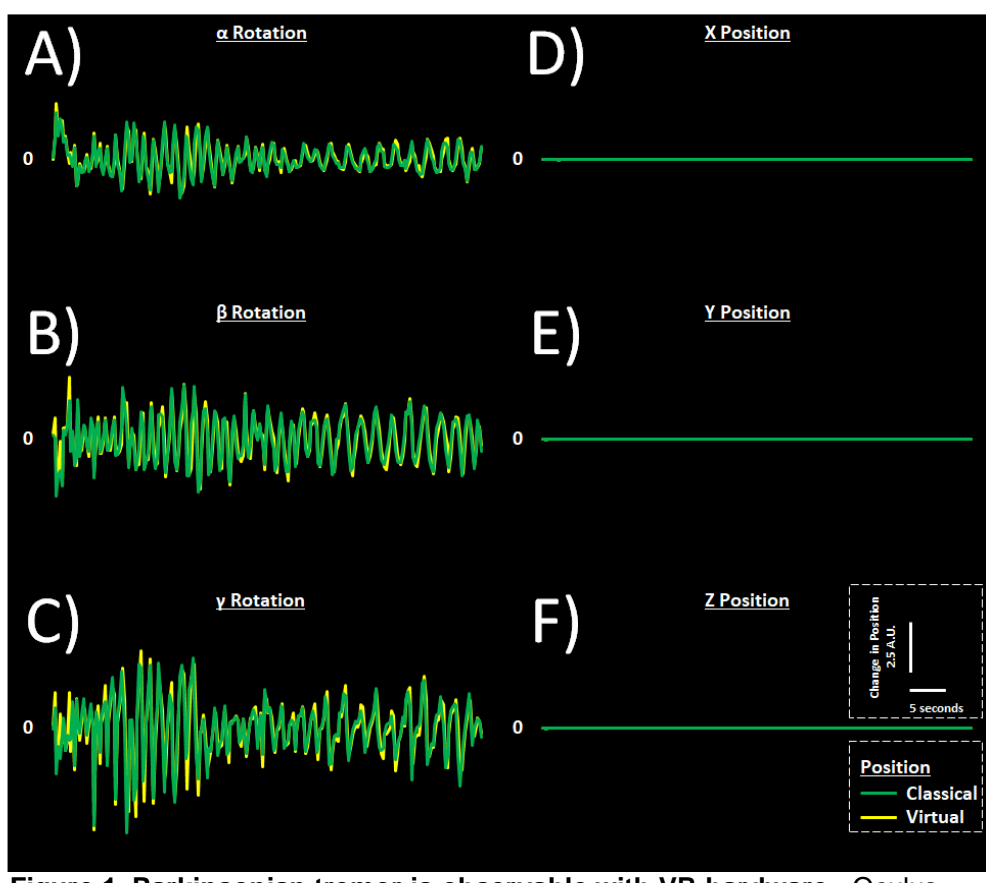

Figure 1. Parkinsonian tremor is observable with VR hardware. Oculus Rift + Touch controller sensory hardware together with Unity software were utilized to record positional data associated with Parkinsonian IHT. Data was captured at $4 \mathrm{~Hz}$ of changes in rotational angles $\alpha, \beta, \gamma(\mathrm{A}-\mathrm{C})$ and Euclidian axes $X, Y, Z$ (D-F). Data of the physical position of controllers is displayed in green and represent participant hands in Classical Reality (CR) (i.e., real world). Data of the real-time position of study participants' digital hands in VR is displayed in yellow. Parkinsonian tremors were more readily observable with rotational data. Data here are of tremor dominant hand of a single representative patient performing a postural tremor test. Low amplitude changes in $\mathrm{X}, \mathrm{Y}, \mathrm{Z}$ coincident with rotational changes were observed in traces of some participants indicating recording in these dimensions is possible.

VR data from patients captured with TSA-OFF showed IHT with high-frequency oscillations (five out of nine patients, Figure 2A, C, E, F, H). These oscillations were notably reduced with TSA-ON in VR. The reductions were instantaneous and persistent indicating robust temporal sensitivity and little algorithmic habituation. Data from a control participant that did not have PD showed no sign of IHT in VR (Figure 2J) and CR (data not shown). Together, these data demonstrate the Oculus Rift as a capable recorder of $\mathrm{IHT}$, that hand rotation caused by tremors provides a robust IHT signature, and that our algorithms eliminate IHT in VR. 
As noted above, we captured IHT signatures separately in VR and CR thus providing an ideal condition with which to test whether reduced IHT in VR results in reduced IHT in CR. As expected, data of CR with TSA-ON retained high-frequency oscillations in most patients (representative patient in Figure 3A). However, CR IHT was notably reduced in one patient (Figure 3B). A deeper inspection of this experiment revealed two interesting observations. The first is that the patient was the youngest in the cohort and had the earliest onset of PD. The second observation is that the patient did not exhibit consistent CR IHT with TSA-OFF (Figure 3B). It is possible that the reduction in CR IHT can occur only in earlyonset PD conditions. Alternatively, it is also possible that the patient was undergoing bouts of CR IHT, and that the time window of the PTT was insufficiently long to capture IHT bouts that would inevitably reoccur. It is also possible that we captured the latent part of postural tremors which are known to be re-emergent (Jankovic, Schwartz and Ondo, 1999)

\section{Method:}

Participants were informed to sign-up for our study via an email newsletter prepared by stoPD, a non-profit organization for PD patients. Participants were not screened for prognosis and severity of $\mathrm{PD}$, or whether on medical or surgical treatment. This study is IRB exempt as experiments are audiovisual in nature (similar to videogames), are brief, harmless, and not physically invasive (see US regulatory code CFR Title 45//section §46.104). All data were collected with participant consent, anonymized during capture, and analyzed in a HIPAA-compliant computational pipeline.

Measurements were recorded with the utmost safety measures, to ensure that our participants had a safe experience.

We employed the Oculus Rift $S$ headset and its Touch controllers (Facebook, Menlo Park, CA). The Oculus was tethered to a laptop running a Unity environment (Unity v2019.1.5f1, San Francisco, CA) for the capture of changes in rotation and Cartesian axes.

The tremor stabilization ideation and algorithms

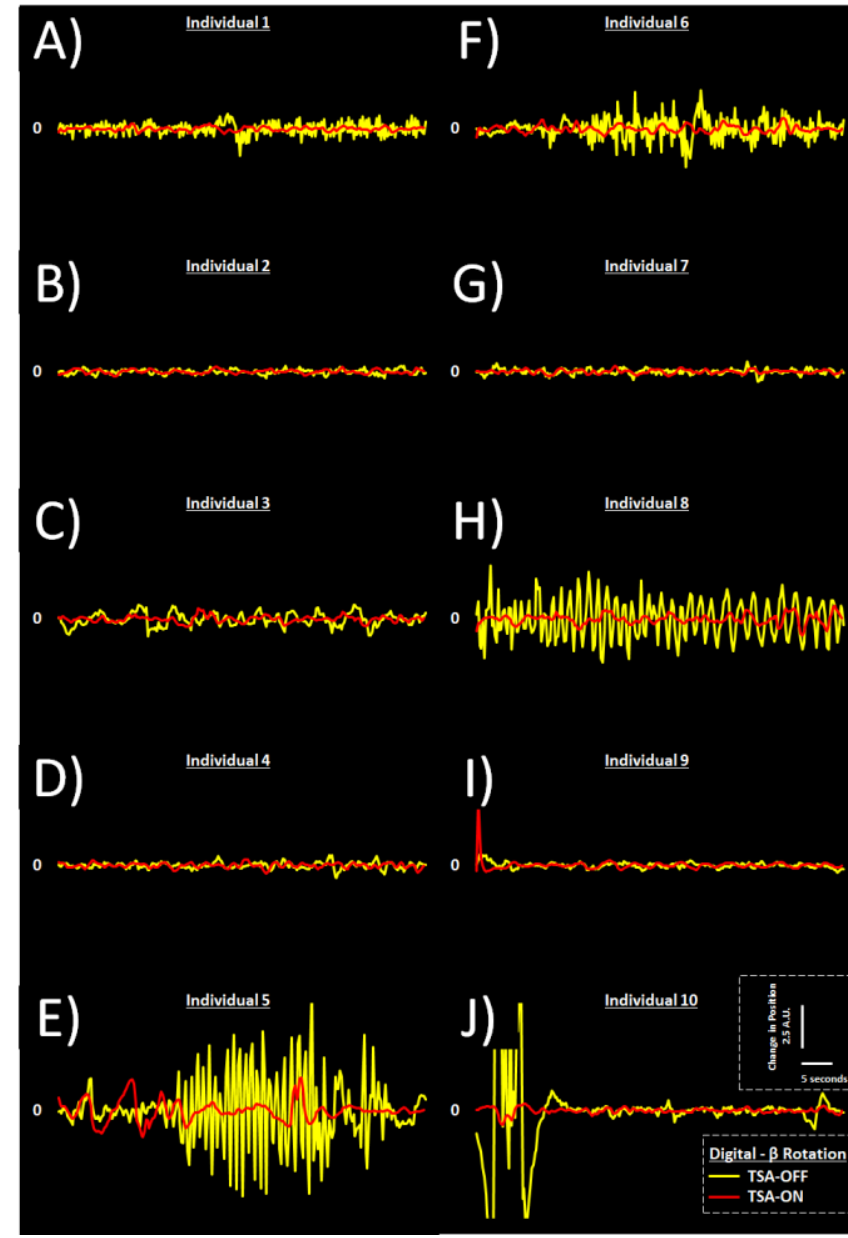

Figure 2. Parkinsonian involuntary hand tremors can be removed in VR. Representative $\beta$ rotational changes of digital hand in VR presented here. Tremor-stabilizing algorithms (TSA) were either turned off (TSA-OFF, yellow) or on (TSA-ON, red) for all patients $(A-I)$ and control participant $(J)$. Only some patients exhibited IHT (A, C, E, F, H). Of these patients, all had IHT notably reduced in VR when tremor stabilization was turned on (TSA-ON). Patients did not undergo screening (see Table 1) thus variability in IHT was expected. High amplitude spikes in control participant trace $(\mathrm{J})$ likely due to whole body movement and does not have high frequency character of Parkinsonian IHT.

we developed here are along the lines of equalizer algorithms from up to six decades ago, well before the age of modern VR (Sutton and Sykes, 1967; Riley and Rosen, 1987; Riviere and Thakor, 1996; Gonzalez et al., 2000). Briefly, our implementation works by capturing the position and rotation of users $\mathrm{CR}$ hands at $200 \mathrm{~Hz}$. At any given moment in time, we employed the lagging $8 \%$ of this data stream to form a moving average centroid that positioned users' digital hands in VR. This mimicked smooth hand motions. For analyses, CR and VR hand positions were stored in data files at $4 \mathrm{~Hz}$. 
Data files were uploaded to a Jupyter Notebook on an AWS EC2 server (Amazon, Seattle, WA). Within the Jupyter environment, data was processed with python using Pandas, Numpy, Scikit-Learn and Scipy libraries. Data was not aggregated for further statistical analyses due to the low sample size of this study.

We observed that digital hands in VR temporarily moved to a new position with activation of tremor stabilization algorithms. This resulted in outlier positions that were identified with scipy.stat.zscore ( $\mathrm{z}$ threshold > 3) and removed with Pandas.

During the PTT, participants often shifted their bodies against the wall. This introduced drift in the data that was removed with functions scipy.signal.detrend and scipy.signal.butterworth which together remove linear trends and low frequency oscillations. The Butterworth function contained a convenience function that centered all data at $y$-axis $=0$.

\section{Discussion:}

Multidisciplinary neurotechnology holds the promise of understanding and non-invasively treating neurodegenerative diseases. In this preclinical trial, we combined neuroscience together with the nascent field of medical virtual reality and generated several important observations. First, we established the Oculus Rift virtual reality system as a potent measurement device for parkinsonian involuntary hand tremors. Interestingly, we determined changes in rotation were the most sensitive marker of PD IHT. Secondly, we determined parkinsonian tremors can be abolished in VR with algorithms that remove tremors from patients' digital hands. We also found that PD patients were interested in and were readily able to use VR hardware and software (data not shown). Together these data suggest PD patients can enter VR and be asymptotic of PD IHT. Importantly, VR is an openmedium where patients can perform actions, activities and functions that positively impact their real lives for instance, one can sign tax return documents in VR and have them printed on real paper or directly sent via internet to government tax agencies. Lastly, we generated a technical framework wherein Classical Reality can be measured side-byside with Virtual Reality. With this framework, we observed anecdotal evidence of parkinsonian tremors being reduced in real life when our algorithms abolished digital hand tremors in VR.

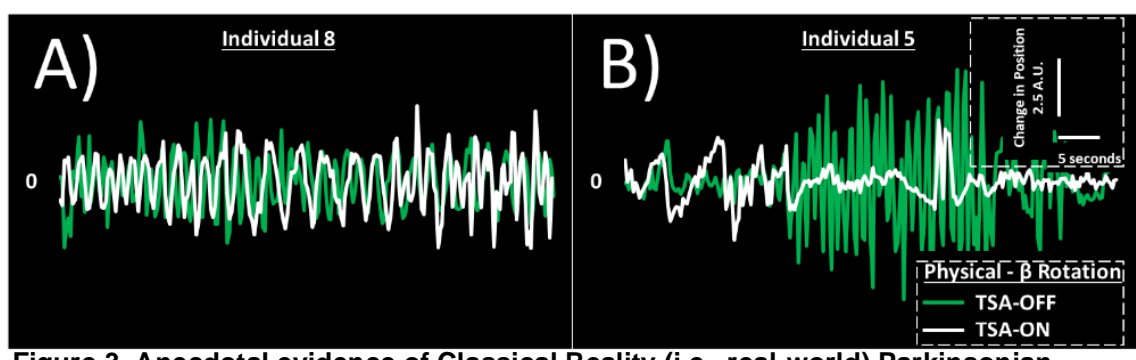

Figure 3. Anecdotal evidence of Classical Reality (i.e., real-world) Parkinsonian tremor reduction associated with VR tremor stabilization. Patients were observing their digital hands in VR with tremors (TSA-OFF) or without tremors (IHT-ON). Data here is of $C R$ changes in patients hand tremors (i.e., real physical world changes).

Representative $\beta$ rotational changes of TSA-OFF are green and TSA-ON are white. Patients exhibiting tremors continued to have IHT in CR regardless of IHT ON or OFF (representative patient shown in (A). However, a single patient with early onset PD had tremors notably reduced during IHT-ON (B).

The exploratory nature of preclinical trials meant our sample size $(n=10)$ was low and was not blinded. Moreover, our $4 \mathrm{~Hz}$ recordings while in the range of typical PD tremor frequency, meant not all tremors were captured (Fourier decomposition; data not shown). Importantly, we did not screen patients for severity of PD prognosis, medical history, or current medical and surgical treatments Despite these addressable shortcomings we established VR as a potent tool for the study of PD. The Oculus Rift we employed cost $\sim \$ 400$ USD at the time of writing. However, the full cost was notably more as it was tethered to custom high-performance computer. The need for such computers is now moot with capable standalone VR systems such as the Oculus Quest. VR systems, in general, are projected to fall to under 
$\$ 100$ USD in the near future suggesting VR can be an accessible, hypersensitive tool for non-invasive studies on PD.

As noted above, we observed and recorded anecdotal evidence of reduced tremors in real life associated with VR with tremor stabilization. We must stress this interesting result must be accompanied with measured restraint as only one of the five patients with tremors in our study exhibited this effect. Further investigation with more patients is needed. If the result holds, our approach has potential as a noninvasive tool to help populations, not limited to PD, but also people afflicted by Essential Tremor, and other movement disorders that make everyday tasks incredibly challenging.

Our work could also further the understanding of Parkinson's disease as theories to explain our observation would have to be explored. One potential theory is tremor stabilization provides an antisignal that restores normal neural activity. Anti-signals can occur in several forms. They could be a train of high-frequency pulses in response to an oscillatory trigger (Rosin et al., 2011; Little et al., 2013) or targeted series of pulses to reset oscillatory circuits (Adamchic et al., 2014). They could also emulate the wild-type character of healthy individuals. Our algorithms remove tremors thus generating an illusion of wild-type hand stability for visual input into central circuits. We believe this presents a discordance of visual-mechanosensory-proprioceptive alignment in subcortical CNS structures responsible for multisensory signal integration. These structures may be thalamic as recent work suggests (Sieben, Röder and Hanganu-Opatz, 2013; Bieler et al., 2018) leaving the possibility of restored 'healthy' incoherence in pathologically synchronous thalamic circuits.

We must note a fundamental difference in anti-signals in the literature relative to our work. These are delivered typically with short electrode pulses that are limited in frequency and amplitude as overstimulation causes unwanted side effects (Beric et al., 2002; Hariz, 2002; Krack et al., 2002; Stefurak et al., 2003; Temel et al., 2006; Tripoliti et al., 2008). Our approach introduced anti-signals based on light and the visual field. This is a paradigm change that likely is characterized by a different ceiling of stimulation. This potential benefit together with the results described here warrant further investigation of our approach of applying virtual reality towards Parkinson's disease therapy.

\section{Acknowledgments:}

Special thanks to Games for Change. To Olivia Goldman and Jared Cameron (NeuroStorm, Inc) for their helpful insight on this work, to Kurt Young (Mokuni Games, LLC) for his inspiring work expanding the potential of medical VR and to Alex Montaldo, Roberta Marongiu (stoPD, Weill Cornell Medicine) and Nisha L Sajnani (NYU) for coordinating participants for this study and for exploring novel ways to tackle Parkinson's disease. Lastly, a posthumous thank you to Jose Zambrano for being an engaging and enlightening part of our development team.

\section{References:}

Adamchic, I. et al. (2014) 'Coordinated reset neuromodulation for Parkinson's disease: Proof-of-concept study', Movement Disorders. doi: 10.1002/mds.25923.

Benabid, A. L. et al. (1994) 'Acute and long-term effects of subthalamic nucleus stimulation of Parkinson's disease', in Stereotactic and Functional Neurosurgery. doi: 10.1159/000098600.

Beric, A. et al. (2002) 'Complications of deep brain stimulation surgery', in Stereotactic and Functional Neurosurgery. doi: 10.1159/000064600.

Bieler, M. et al. (2018) 'Multisensory integration in rodent tactile but not visual thalamus', Scientific 
Reports. doi: 10.1038/s41598-018-33815-y.

Buhmann, C. et al. (2017) 'Adverse events in deep brain stimulation: A retrospective long-term analysis of neurological, psychiatric and other occurrences', PLOS ONE. doi: 10.1371/journal.pone.0178984.

Cools, R. et al. (2003) 'L-Dopa medication remediates cognitive inflexibility, but increases impulsivity in patients with Parkinson's disease', Neuropsychologia. doi: 10.1016/S0028-3932(03)00117-9.

Cyron, D. (2016) 'Mental side effects of deep brain stimulation (DBS)for movement disorders: The futility of denial', Frontiers in Integrative Neuroscience. doi: 10.3389/fnint.2016.00017.

Eusebio, A. et al. (2011) 'Deep brain stimulation can suppress pathological synchronisation in parkinsonian patients', Journal of Neurology, Neurosurgery and Psychiatry. doi:

10.1136/jnnp.2010.217489.

Gonzalez, J. G. et al. (2000) 'Optimal digital filtering for tremor suppression', IEEE Transactions on Biomedical Engineering. doi: 10.1109/10.841338.

Hariz, M. I. (2002) 'Complications of deep brain stimulation surgery', Movement Disorders. doi: $10.1002 /$ mds.10159.

Hickey, P. and Stacy, M. (2016) 'Deep brain stimulation: A paradigm shifting approach to treat Parkinson's disease', Frontiers in Neuroscience. doi: 10.3389/fnins.2016.00173.

Jankovic, J., Schwartz, K. S. and Ondo, W. (1999) 'Re-emergent tremor of Parkinson's disease', Journal of Neurology Neurosurgery and Psychiatry. doi: 10.1136/jnnp.67.5.646.

Koller, W. C., Vetere-Overfield, B. and Barter, R. (1989) 'Tremors in early Parkinson's disease', Clinical Neuropharmacology. doi: 10.1097/00002826-198908000-00006.

Krack, P. et al. (2002) 'Postoperative management of subthalamic nucleus stimulation for parkinson's disease', Movement Disorders. doi: 10.1002/mds.10163.

Kühn, A. A. et al. (2008) 'High-frequency stimulation of the subthalamic nucleus suppresses oscillatory $\beta$ activity in patients with Parkinson's disease in parallel with improvement in motor performance', Journal of Neuroscience. doi: 10.1523/JNEUROSCI.0282-08.2008.

Lee, H. J. et al. (2016) 'Tremor frequency characteristics in Parkinson's disease under resting-state and stress-state conditions', Journal of the Neurological Sciences. doi: 10.1016/j.jns.2016.01.058.

Lee, S. Y. et al. (2016) 'Activities of daily living questionnaire from patients' perspectives in Parkinson's disease: A cross-sectional study', BMC Neurology. doi: 10.1186/s12883-016-0600-9.

Little, S. et al. (2013) 'Adaptive deep brain stimulation in advanced Parkinson disease', Annals of Neurology. doi: 10.1002/ana.23951.

Marras, C. et al. (2018) 'Prevalence of Parkinson's disease across North America', npj Parkinson's Disease. doi: 10.1038/s41531-018-0058-0.

Mirelman, A. et al. (2016) 'Addition of a non-immersive virtual reality component to treadmill training to reduce fall risk in older adults (V-TIME): a randomised controlled trial', The Lancet. doi: 10.1016/S01406736(16)31325-3.

Nabi, A. et al. (2013) 'Minimum energy desynchronizing control for coupled neurons', Journal of Computational Neuroscience. doi: 10.1007/s10827-012-0419-3. 
Pringsheim, T. et al. (2014) 'The prevalence of Parkinson's disease: A systematic review and metaanalysis', Movement Disorders. doi: 10.1002/mds.25945.

Riley, P. O. and Rosen, M. J. (1987) 'Evaluating manual control devices for those with tremor disability', Journal of rehabilitation $R \& D$.

Riviere, C. N. and Thakor, N. V. (1996) 'Modeling and canceling tremor in human-machine interfaces', IEEE Engineering in Medicine and Biology Magazine. doi: 10.1109/51.499755.

Rosin, B. et al. (2011) 'Closed-loop deep brain stimulation is superior in ameliorating parkinsonism', Neuron. doi: 10.1016/j.neuron.2011.08.023.

Salat, D. and Tolosa, E. (2013) 'Levodopa in the treatment of Parkinson's disease: Current status and new developments', Journal of Parkinson's Disease. doi: 10.3233/JPD-130186.

Sieben, K., Röder, B. and Hanganu-Opatz, I. L. (2013) 'Oscillatory entrainment of primary somatosensory cortex encodes visual control of tactile processing', Journal of Neuroscience. doi:

10.1523/JNEUROSCI.4432-12.2013.

Stefurak, T. et al. (2003) ‘Deep brain stimulation for Parkinson's disease dissociates mood and motor circuits: A functional MRI case study', Movement Disorders. doi: 10.1002/mds.10593.

Sutton, G. G. and Sykes, K. (1967) 'The effect of withdrawal of visual presentation of errors upon the frequency spectrum of tremor in a manual task', The Journal of Physiology. doi:

10.1113/jphysiol.1967.sp008208.

Syrkin-Nikolau, J. et al. (2018) 'Coordinated reset vibrotactile stimulation shows prolonged improvement in Parkinson's disease', Movement Disorders. doi: 10.1002/mds.27223.

Temel, Y. et al. (2006) 'Behavioural changes after bilateral subthalamic stimulation in advanced Parkinson disease: A systematic review', Parkinsonism and Related Disorders. doi:

10.1016/j.parkreldis.2006.01.004.

Tripoliti, E. et al. (2008) 'Effects of contact location and voltage amplitude on speech and movement in bilateral subthalamic nucleus deep brain stimulation', Movement Disorders. doi: 10.1002/mds.22296.

Weaver, F. M. et al. (2009) 'Bilateral Deep Brain Stimulation vs Best Medical Therapy for Patients', JAMA.

Wilson, D. and Moehlis, J. (2015) 'Clustered Desynchronization from High-Frequency Deep Brain Stimulation', PLoS Computational Biology. doi: 10.1371/journal.pcbi.1004673.

Wingeier, B. et al. (2006) 'Intra-operative STN DBS attenuates the prominent beta rhythm in the STN in Parkinson's disease', Experimental Neurology. doi: 10.1016/j.expneurol.2005.09.016. 